\title{
Wireless power transmission system for charging the inspection robot on power transmission lines
}

\author{
Mingbo Yang, Qingzeng Ma, Guodong Yang, Zize Liang, Hao Lin \\ State Key Laboratory of Management and Control for Complex Systems, \\ Institute of Automation, Chinese Academy of Sciences, \\ Beijing 100190, P. R. China; \\ jlsthsdqyx@sina.com \\ \{Qingzeng.Ma, Guodong.yang, Zize.liang, Hao.lin\}@ia.ac.cn
}

\begin{abstract}
In order to prolong continuous working time of the mobile robot to inspect on transmission lines, a robotic power management system is introduced in this paper based on the wireless power transmission technology. Magnetic resonance coupled WPT (wireless power transmission) unit is applied and its performance is analyzed in form of electrical circuit mode method. By modeling the output power of WPT unit, the relationship between coupled coefficient, angular frequency and amount of output power is given, and furthermore the energy consumption of each unit in power management system is quantified. Experiment results verified the validity of the circuit model of WPT and showed that the system could work conveniently in wireless way to provide power for the inspection robot.
\end{abstract}

Keywords-wireless power transfer, coupled coefficient, Q-factor

\section{INTRODUCTION}

Inspection robot for power transmission line is gradually putting into use now because heavy inspection task for power transmission lines urges electric utilities to put inspection robot into practice. Researchers have been working on designing one kind of mobile robots to partly or fully perform the inspection tasks of power transmission lines ${ }^{[1][2]}$. But, there is still a problem which has serious influence on performance of inspection robot-the continuous working period of robot. Most inspection robot uses battery pack as power source. Because of the large amount of power need by motors and control system, the robot could not work for long time and often need the maintenance. Not too much effective means are provided in relative researches. Reference 3 provides an induction power system; it uses induction voltage to drive the robot directly, if the robot is designed to walk on ground line of power transmission lines, it induction method doesn't work anymore because there is no chance for the robot to induce power from power transmission line. So, we designed a charging dock which is built on the tower of transmission lines to induce power from the mother line and transmit it to the robot hanging up on the ground line. Magnetic resonance wireless power transmission method is used here to transfer power wirelessly, and the coils used for WPT is equipped on both the robot and the charging dock.

Magnetic resonance coupling technology has received more attention than other wireless power transfer technologies recently due to its capability to transfers energy across large air gap with high efficiency. Although the coupled resonance based WPT need much more attention to design and test the drive circuit as its working frequency is usually up to several mega $\mathrm{Hz}$, it's effective working distance which is up to several meters (decides by the material and the size of the resonance coil in application) is still really attractive for medium distance wireless power transmission applications. In this paper, the fundamental characteristics of a special magnetic resonance system for transmission line inspection robot have been examined. Equivalent circuit is given in order to give numerical analysis of the resonance system. In order to test the output power of the WPT unit, current in load coil is calculated, and the relationship between coupled coefficient, angular frequency and output power is given. Power transmission ratio is also given in chart which shows that amount of power loss of WPT accounts for few part of the total loss of the power management system and the ratio is acceptable.

Equivalent circuit are also verified by experiment which shows that the proposed method is suitable for charging the inspection robot and the coupled resonance wireless power transmission system could also be applied in many other fields to avoid the trouble or obstacle of using electric cord.

A wireless power transmission technique based robotic power management system is introduced: Section 2 shows the general function charts of the power management system, and especially the wireless power transmission unit of the system. Section 3 details the circuit model of WPT for calculating the output power of this unit; Section 4 introduces the experiments results and proves that the WPT based power management system can efficiently transmit power wirelessly and the design in this paper has the function of prolonging continuous working period of the inspection robot.

\section{OVERVIEW OF THE SYSTEM}

Main units of the WPT based power management system is presented in Fig.1. As shown in the figure, the system consists of induction unit, wireless power transmission unit and inspection robot. The transmitter unit and receiver unit placed in upper dashed frame behave as WPT system's input and output port, transmitter unit is equipped on induction unit and receiver unit is equipped on inspection 
robot. The induction unit induce power from the power transmission line by a special current transformer ${ }^{[4]}$, it belongs to charging dock fixed in magnetic field of the transmission line and outputs induction power for transmit unit. Transmitter unit and receiver unit could be conceived as LC resonance tanks and they transmit power wirelessly when they are tuned to oscillate at the same frequency. Then inspection robot gets power from the receiver unit and charges its batteries.

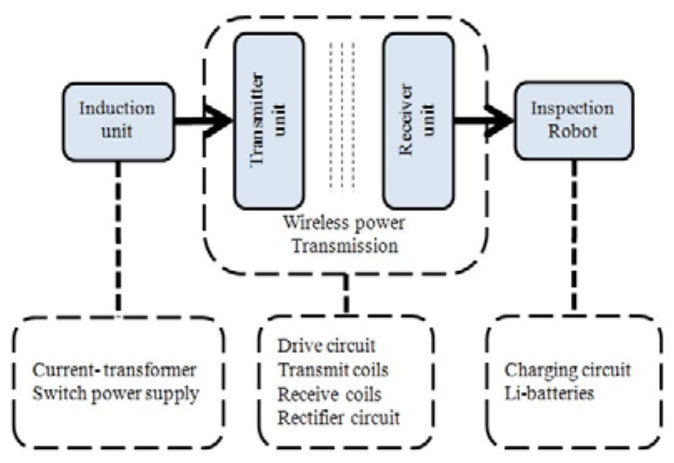

Fig.1 WPT based power management system

Induction unit derive power from power transmission line by using special current transformer as mentioned before, switch power supply circuit modulate the power into DC power by $13.8 \mathrm{~V}$ for drive circuit, the drive circuit which belongs to wireless power transfer unit generate sinusoidal signal and amplify the signal into power exciting signal for wireless power transmission system. The WPT system consist mainly two parts, the transmitter unit and the receiver unit. Drive circuit and transmit coils made up of the transmitter unit. The receive coils and rectifier circuit made up of the receiver unit. Receiver unit output another $48 \mathrm{~V}$ DC power signal for charging li-battery packs on inspection robot.

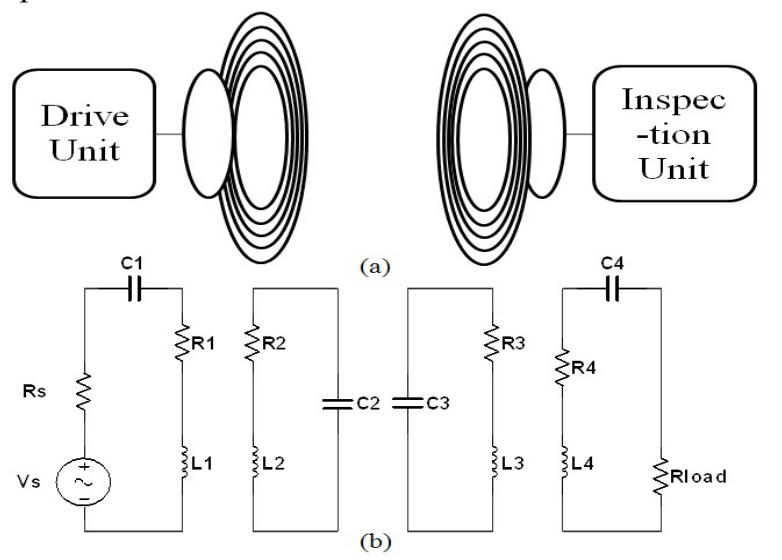

Fig.2 (a) Simplified schematic of the system;

(b) Electrical model of WPT

\section{MAGNETIC RESONANCE WPT UNIT}

Couple mode theory [5] has been originally used to describe resonance-based coupling [6], [7]. It is not convenient for us to model and calculate parameters of the system using CMT theory because if we want to measure or test of the input or output of the physical system, CMT is helpless. So, we need other method to describe the system and help us to analyze the coupled system. As a simple circuit-based model for the coupled systems is presented in [8], and the effect of the low-factor and the low coupling between the source and load coils can be compensated by using high Q-factor coils, so we utilize the circuit theory and induction theory to describe the system which is actually built by using copper wire as drive coils and resonance coils.

Fig. 2 (a) shows the simplified chart of WPT unit of the power management system including the drive unit, the high-Q coils and the inspection unit which plays the role of load here. There are four coils here to realize efficient power transfer, and they are referred to as driver, primary, secondary, and load coil (denoted as coils 1 to 4). Here, primary coil and secondary coil is shape of planar spiral coil because it would be space-saving than that of the air cored solenoid. Besides, in order to tune the two planar spiral coils in to state of resonance, we use air capacitance to change the resonance frequency of each coil, but it is not shown in Fig.2 (a). Fig. 2 (b) shows the simplified schematic and electrical model of the four-coil system. By applying circuit theory to this system, the relationship between current running in each coil and the voltage of power source applied to the driver coil can be expressed in the following equations set:

$$
\left\{\begin{array}{l}
\dot{I}_{1} Z_{1}+j \omega \dot{I}_{2} M_{12}=V_{s} \\
\dot{I}_{2} Z_{2}+j \omega\left(\dot{I}_{1} M_{12}-I_{3} M_{23}\right)=0 \\
\dot{I}_{3} Z_{3}+j \omega\left(\dot{I}_{4} M_{34}-\dot{I}_{2} M_{23}\right)=0 \\
\dot{I}_{4} Z_{4}+j \omega \dot{I}_{3} M_{34}=0
\end{array}\right.
$$

Where, $I_{i}$ means the current in coil $i, V_{s}$ is the amplitude of voltage source applied to the driver coil, $Z_{i}$ is the sum of effective resistance, inductance, and capacitance of the coil $i, M_{m n}$ is the mutual inductance between coil $n$ and $m$,

$$
M_{m n}=k_{m n} \sqrt{L_{m} L_{n}}
$$

Where the coupled coefficient between coil $m$ and $n$ is denoted as $k_{m n}$. Primary coil and secondary coil have the same structure which is made up of planar spiral coil in series with air capacitance. So it performs as LC circuit with low impedance comes from copper wire. The system could only work on state of LC resonance, and only the power of the resonance frequency can pass this four coil wireless path. In the view of the whole system, the four coils WPT is a dual port network with LC circuit as input port and output port respectively which is working on 
resonance state, so at this time, the inductance reactance and capacitance reactance of the coils cancel out each other. The two LC resonant body show only resistance character which means that value of impedance of input port and output port of the dual port network only has real part. Besides, not only the primary coil and the second coil of WPT system need to be on resonance state, the drive coil and the load coil should also be in resonance state. Resonance of four coils at the same time is vital for four-coil WPT to transfer power effectively. Then, to solve the equation $1, Z_{i}$ can be replaced by $R_{i}$, shown as formula (3)

$$
\left\{\begin{array}{l}
Z_{1}=R_{s}+R_{1} \\
Z_{2}=R_{2} \\
Z_{3}=R_{3} \\
Z_{4}=R_{\text {load }}+R_{4}
\end{array}\right.
$$

Then we can deduce $\dot{I}_{4}$

as

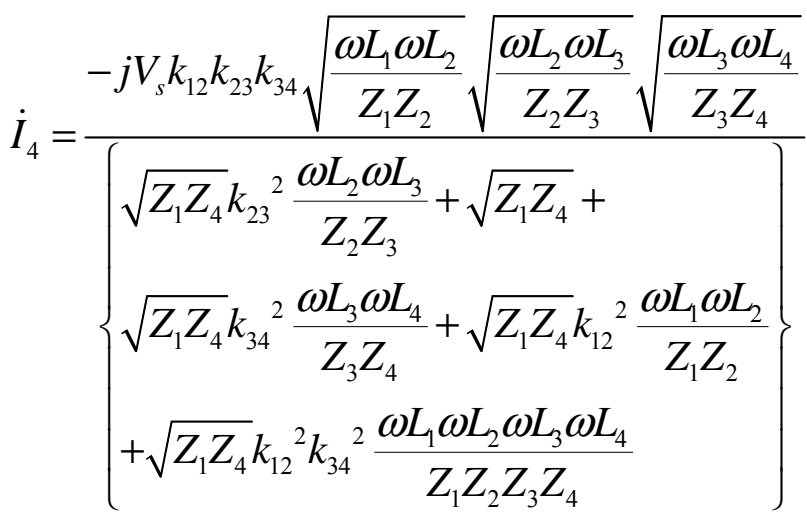

Formula 4 shows that $\dot{I}_{4}$ is 90 degree lag in phrase compared to input power signal and the amplitude of current is strongly affected by item- $\frac{\omega L}{Z}$, which is $\mathrm{Q}$ of each coils.

Then output power of the system could be deduced as

$$
\begin{aligned}
& P_{\text {out }}=\left|\dot{I}_{4}\right|^{2} R_{\text {load }}= \\
& \frac{\omega^{6}\left(k_{12} k_{23} k_{34}\right)^{2} L_{1} L_{2}{ }^{2} L_{3}{ }^{2} L_{4} V_{s}^{2} R_{\text {load }}}{\left[\begin{array}{l}
Z_{1} Z_{4} k_{23}{ }^{2} L_{2} L_{3} \omega^{2}+Z_{1} Z_{2} Z_{3} Z_{4}+Z_{1} Z_{2} k_{34}{ }^{2} L_{3} L_{4} \omega^{2} \\
+k_{12}{ }^{2} L_{1} L_{2} Z_{3} Z_{4} \omega^{2}+k_{12}{ }^{2} k_{34}{ }^{2} L_{1} L_{2} L_{3} L_{4} \omega^{4}
\end{array}\right.}
\end{aligned}
$$

The output power of the WTP could be plotted as Fig.3. This plot shows output power magnitude as a function of frequency $\omega$ and coupling coefficient $k_{23}$. Here we can see that when the frequency is about $6.3 \mathrm{MHz}$ (this frequency point could be easily changed when each distance of the four coils is changed or the input power is increased or lowered, and those factors and its performance has been analyzed in detail in another paper, so we ignore it here), the system could get into the ideal resonance coupled working state because at this point, there is power output when coupled coefficient is really small. As coupled coefficient $k_{23}$ increases from 0.0001 to 0.05 in the figure, output power can be divided into to parts. At the beginning, the maximum output power occurs at the single angular frequency $\omega_{0}$, and it increases as coupled coefficient $k_{23}$ increased, this region is called weak coupled area; when the coupled coefficient keep on increasing, single frequency at which the maximum output power is achieved turns to double frequencies $\omega_{1}$ and $\omega_{2}$, and $\Delta \omega=\left|\omega_{1}-\omega_{2}\right|$ is increasing. This region is call strong coupled region.

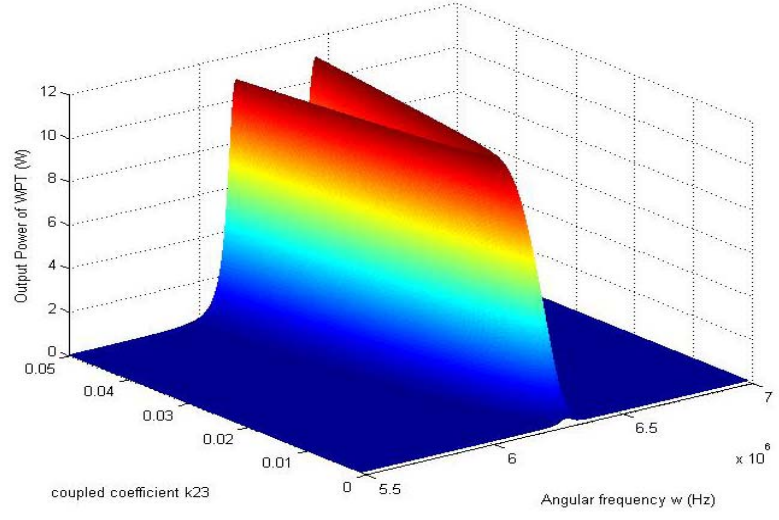

Fig.3 Output power of the magnetic coupled system

It is obviously that the ideal point of $\omega_{0}$ is the critical point between weak coupled area and strong coupled area because at this critical point, the system could transfer the maximum power at the maximum distance. That is the principle and essence of WPT. On top of the figure there are two ridges and divided toward respectively to higher and lower frequencies each, so the frequency where the maxim output power could be achieved is changing as coupled coefficient $k_{23}$ is increasing. Coupled coefficient $k_{23}$ has great relationship with distance between primary coil and second coil and if $k_{23}$ is smaller than the critical point of frequency (here it is almost 0.02), the system could not output the maximum power at that distance as that of strong coupled mode.

In order to improve output current in this model, using high-Q coils is necessary because the efficiency of WPT system has great relationship with the $\mathrm{Q}$ value of resonance 
coils, from formula 4 , substitute the item $\frac{\omega L}{Z}$ with $\mathrm{Q}$, then power transferring efficiency of the resonance coils with Q-factor vary from 0 to 500 on fixed operating range $d_{23}$ as of $40 \mathrm{~cm}$ is plotted as Fig.4 (a), it's obvious that if Q of coil is less than 50, power transferring efficiency is low and the curve is steep, so using high Q coils is necessary; Besides, if the Q of the coils is fixed(in this case, it is set as 300), the efficiency of resonance coils with operating range $d_{23}$ vary from 0 to $150 \mathrm{~cm}$, power transmission efficiency can be plotted as Fig.4 (b), which shows that efficiency rises first and fall down after it reaches the highest point at the distance of $46 \mathrm{~cm}$, efficiency could up to $68 \%$ at this critical distance, so operation range $d$ should be carefully set when transmitting power with coupled resonance method.

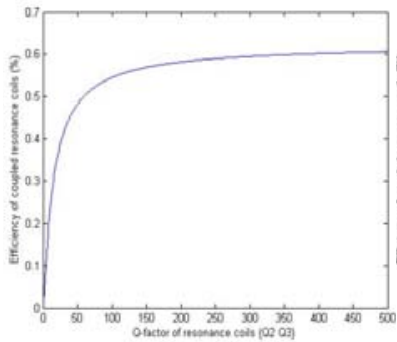

(a)

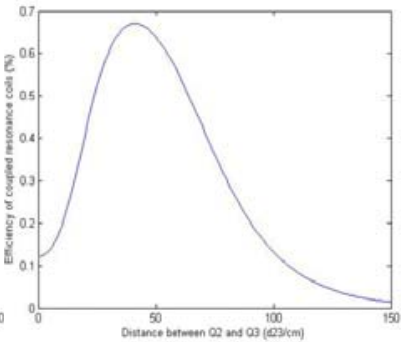

(b)
Fig.4 Efficiency with $\mathrm{Q}_{2}, \mathrm{Q}_{3}$ and $\mathrm{d}_{23}(\mathrm{~cm})$

\section{EXPERIMENTS}

To improve the model and the calculation result above, experiment on power transfer has been implemented. Induction unit consist current transformer, DC converter, sine wave generator, wide band microwave amplifier and PIC controller. Output impedance of the amplifier is $50 \Omega$. In order to test and supervise the output power of the induction unit and also the standing wave going into the amplifier, a power meter and standing meter for microwave is used in series connection with the drive coil. So we can monitor the amount of output power and the returning power to keep the wide band microwave power amplifier from being overloaded or damaged by voltage of standing wave. After power on the system, increasing the amplitude of sine wave generator output could increase the output power of amplifier. Then magnetic field intensity of primary coil is increased and the resonance strength between primary coil and second coil is increased.

Fig. 5 shows Energy distribution of Power management system. In this experiment, the maximum power loss occurs in drive unit which is nearly $35 \%$ of the main output of current transformer, power loss of resonance coupled of the two high Q coils is nearly $13 \%$ of the whole output of current transformer, the distance $d_{23}$ is $65 \mathrm{~cm}$ in the picture, and output power is $6 \mathrm{~W}$ when input power is $18.32 \mathrm{~W}$. So to decrease the loss in induction unit is another task for the system to be applied in practice. Fig. 6 shows the system powering a $6 \mathrm{~W}$ bulb with WPT system at the distance of 65 $\mathrm{cm}$.

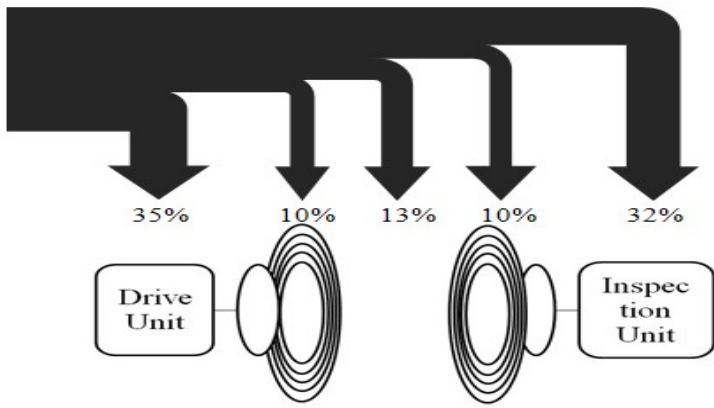

Fig.5 Energy distribution of Power management system

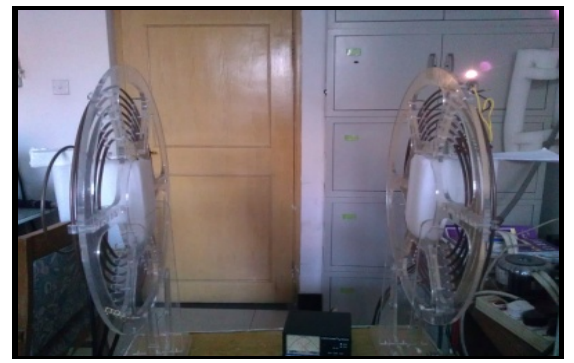

Fig.6 Energy distribution of Power management system

\section{CONCLUSION}

In this paper, we presented a resonance coupled based wireless power management system for power transmission line inspection robot. Experiment results match well with the calculating result and at the distance of $65 \mathrm{~cm}$, the system can transmit power at the efficiency of more than $30 \%$ and it performs to be applicable for charging our inspection robot wirelessly.

\section{ACKNOWLEGEMENT}

This work is supported by the National High Technology Research and Development Program of China (863 Program) (2011AA04A104)

\section{REFERENCES}

11] Jun Sawada, Kazuyuki Kusumoto, Yasuhisa Maikawa, Tadashi Munakata, Yoshinobu Ishikawa. A Mobile Robot For Inspection Of Power Transmission Lines. IEEE Transactions on Power Delivery, Vol. 6, No.1, January 1991.

[2] Hector Beltran, San Segundo, Vicente Fuster, Lourdes Perez, Pedro Mayorga. Automated Inspection of Electric Transmission Lines:The power supply system. 1-4244-0136-4/06. IEEE, 2006

[3] S.Peungsungwa1, B. Pungsiri, K. Chamnongthai and M. Okuda. Autonomous robot for a powertransmission line. The 2001 IEEE International Symposium on. 2001 , Page(s): 121 - 124 vol. 2I. S. Jacobs and C. P. Bean, "Fine particles, thin films and exchange anisotropy," in Magnetism, vol. III, G. T. Rado and H. Suhl, Eds. New York: Academic, 1963, pp. 271-350.

[4] Mingbo Yang, Zize Liang, En Li, Kailiang Zhang, Guodong Yang. Design of current transformer for power transmission lines inspection robot. Material Science and Engineering Technology Volume:462, Page(s):860-865. 2012

[5] H. Haus, W. Huang. Coupled-mode theory. Proc.IEEE, vol.79, no.10. pp.1505-1518,Oct.1991. 
[6] A.Karalis, J.Joannopoulos, M. Soljacic. Effcient wireless non-radiative mid-range energy transfer. Ann. Phys., vol. 323,pp.34-48,Apr.2007.

[7] A.Kurs, A.Karalis, R.Moffatt, J.D.Joannopoulos, P.Fisher, M.Soljacic. Wireless power transfer via strongly coupled magnetic resonances. Sci.Expr., vol.317,pp.83-86,Jul.2007.

[8] A.Kumar,S.Mirabbasi, M.Chiao. Resonance-based wireless power delivery for implantable devices. in Proc. IEEE Biomedical Circuits and Systems Conf.,pp.25-28. Nov. 26-28, 2009. 